\title{
Expectations for User Experience in Haptic Communication with Mobile Devices
}

\author{
Jani Heikkinen \\ Tampere University of Technology, \\ Human-Centered Technology \\ Korkeakoulunkatu 6 \\ 33720 Tampere, Finland \\ jani.heikkinen@tut.fi
}

\author{
Thomas Olsson \\ Tampere University of Technology, \\ Human-Centered Technology \\ Korkeakoulunkatu 6 \\ 33720 Tampere, Finland \\ thomas.olsson@tut.fi
}

\author{
Kaisa Väänänen-Vainio-Mattila \\ Tampere University of Technology, \\ Human-Centered Technology \\ Korkeakoulunkatu 6 \\ 33720 Tampere, Finland \\ kaisa.vaananen-vainio- \\ mattila@tut.fi
}

\begin{abstract}
The haptic modality - the sense of touch - is utilized very limitedly in current human-computer interaction. Especially in mobile communication, the haptic modality could provide a means for richer multimodal and emotional communication between users over distance. Haptic user interface prototypes have been developed but their user experience has not been studied extensively. We conducted seven focus group sessions to study users' expectations for user experience of haptic interaction, specifically focusing on mobile communication. The paper presents the user experience factors that were regarded by potential users as salient in haptic interaction: subjective quality of the haptic stimuli, privacy, intimacy and spontaneity. Also the possibilities, restrictions and suitability of the haptic mobile communication are discussed. Most appropriate use cases for haptic communication were found to be conveying emotions and binary information. As the main conclusion, we present design guidelines for haptic mobile communication that were drawn up based on the findings.
\end{abstract}

\section{Categories and Subject Descriptors}

H.1.2 [Information Systems]: User/Machine Systems - Human factors; H.5.2 [Information Systems]: User Interfaces - Haptic I/O

\section{General Terms}

Human Factors, Experimentation, Design

\section{Keywords}

Mobile, haptics, communication, guidelines, user experience, focus group study

\section{INTRODUCTION}

The interest in utilizing haptic modality in user interfaces is growing, especially in mobile communication domain. The term haptic can be defined as "Relating to or based on the sense of

Copyright is held by the author/owner(s).

MobileHCI'09, September 15 - 18, 2009, Bonn, Germany.

ACM 978-1-60558-281-8. touch" [18]. Haptic interaction can be used, for example, in teleoperation of devices or in applications for users with impaired vision. By haptic mobile communication we mean information exchange by sending and receiving haptic information between two people with hand-held mobile devices. The haptic stimulus, for example vibration, pressure, skin stretch or temperature, is created by touching the device or by making gestures with it. Also, the use of mobile devices with small displays can be enhanced with multimodal interaction. By representing certain elements of graphical user interfaces via haptic modality, cognitive load can be reduced and the use of mobile devices can become more efficient [7],[12],[14],[16]. Our interest, however, lies in the new opportunities haptic interaction can bring to mobile communication.

Mobility has enabled new ways of communication and people often do mobile communication simultaneously with other tasks in diverse contexts. These situations are challenging and complex as the tasks are competing for users' limited attention. The most cognitively demanding mobile situations are the ones requiring visual and motor tasks simultaneously [20]. As mobile devices must be small to carry around, they have small displays even though their use is heavily based on our visual system. As a result, they compete for vision with other tasks, such as observing our surroundings. By representing information via the haptic instead of visual modality, there is an opportunity to lower the need for users' cognitive resources in such use contexts.

Current remote communication applications rely mostly on audio or text to convey information, while in fact people's communication relies more on non-verbal modalities. It has been estimated that our verbal communication conveys only $7 \%$ of the transferred information, the rest coming from vocal changes and body language [19]. This non-verbal information is often interpreted subconsciously, without our active attention. Most current remote communication methods are not designed to convey non-verbal information and thus, the majority of the information is lost when these methods are used. Therefore, the absence of small, subconscious nuances in mobile communication is a challenge. Information expressed by facial expressions and body language is lost with current mobile communication methods. This is especially true in text-based communication, in which emoticons have become very popular ways to enrich the message and ease the interpretation. While factual information is usually straightforward to communicate by only using auditory or textual modality, emotions are difficult to express. Finding the 
right words can be challenging and the message can be easily misinterpreted when the nuances are lost.

Chang et al. [4] have listed design parameters affecting haptic communication. The parameter "data transfer" classifies the communication as 1) synchronic (requires turn-taking) or 2) asynchronic (independent from the other end and at any time). Thus, the content of the conveyed data can be either continuous or discrete.

Haptic modality includes elements that make it an intriguing research topic. It is very social and private: touching is a strong social message and is not used with everyone in face-to-face communication. The sense of touch offers different ways to code information, e.g. temporal and spatial parameters as well as temperature and pressure. It can be precise and people can feel very slight changes in the properties of material through touch, but the sensations are not absolute (i.e. they are difficult to memorize and describe exactly). Touch is always a two-way sensation between humans and without technology support it always requires a close proximity between people. [17]

The use of haptic modality in interpersonal communication has the potential to improve the user experience of mobile communication devices and services. However, the possibilities and restrictions of haptics are not properly understood. While the existing research in this field is often technology-centered, describing systems for creating haptic stimuli, we took a usercentered and explorative approach to study users' needs and expectations to haptic communication and its user experience. Uniting the research in user experience and haptics is a novel approach, which will increase understanding of human factors to provide more successful haptic applications.

Generally, in HCI literature user experience is considered to cover all the aspects of end-users' interaction with a system. The prior expectations for the experience play a major role in determining the overall satisfaction or dissatisfaction, joy or unhappiness, and benefits or disadvantages of the eventual experience. Therefore, it is crucial to understand potential users' present expectations for the technology and its implementation. We addressed this research challenge by conducting altogether seven focus groups with various types of users. Our aim was to find out how the ability to send haptic information could bring new richness to non-verbal mobile communication.

The paper is structured as follows. First, the related work of haptic technologies and systems, and user experience are presented. Next, the methodology of our focus group study is described. Then, the results concerning users' expectations to haptic user experience are presented. Finally, we discuss the impact of the results and present design guidelines for haptic mobile communication.

\section{RELATED WORK}

We first present related research on systems and prototypes that utilize gestures or other haptic interaction. Then, we briefly discuss the concept and theories of user experience and the various factors that have an influence on it.

Linjama \& Kareesoja [15] present an approach to studying haptic gestures in mobile devices. They demonstrate how simple gestures aided by haptic feedback could be used in a mobile domain. As an input gesture they use a simple tap with a finger and a matching tactile "bump feedback". An application that simulates the movements of a ball using these input and output methods is demonstrated.

Brown and Williamson [3] propose a new concept of communication by using non-verbal sounds and tactile feedback. Their concept system identifies four different gestures and associates a nonverbal sound with the discrete messages. These discrete tactile messages are called tactons or tactile icons [11]. Hoggan and Brewster [11] found no statistically significant differences in recognition rates of tactons combined with audio in mobile (walking) versus stationary situations. However, the participants were trained in neither the auditory nor the tactile icons. Also, they found that information learnt in one modality can be recognized via another modality at a similar rate. Paakkanen et al. [21] similarly studied the perception of haptic stimulus while biking versus being stationary. However, they found significant differences in reaction times and perception accuracy between the setups. In stationary setups, stimuli in the back, leg and wrist areas were easier to recognize while the chest had higher ratings while mobile. Reaction times were overall lower in the stationary setup versus the mobile.

Mediated touch shares the same level of privacy as real touch in a social context as shown by Haans et al. [8]. Touch sensations created by actuators were as intimate as normal touching - body locations, such as the stomach, were as sensitive locations for mediated touch as well as real touch. This suggests that artificial touch has the potential to convey the same emotional information as real touch, which is promising for haptic emotional communication.

Chang et al. [4] have also developed a communication device that takes advantage of the sense of touch. Their ComTouch concept is an attachment to mobile phones providing tactile feedback. For possible use cases they list private and emotional communication, multiplexing information and users with special needs. They evaluated their prototype with users in a multimodal context together with speech. They found three different use-strategies for asymmetric and continuous tactile feedback with speech: an emphasis on phrases or syllables, markers for turn-taking and mimicking patterns to communicate that they are listening.

Smith et al. [22] found that a haptic channel can be used to convey emotive information remotely, although it is not thoroughly understood. They tested a few interaction models for mapping the haptic input and output to a single-degree haptic knob. The relationship between the participating pairs affected the model. Interestingly, the couples mostly preferred the interaction model estimated to be more intimate, while strangers preferred the low intimacy model. Brave et al. [1] also received comments on their early haptic communication device prototype saying it would be suitable for people in a relationship.

Yonahan et al. [23] developed an animal-like haptic device ("hapticat"), which was used to study touch and affect. The device has four features that can be individually controlled: breathing, ears, purring and warming. Participants in their user study were able to recognize three out of five of the hapticat's states (e.g. happy, upset) correctly. The other states are possibly recognizable after refining the action/response mappings to match participants' expectations more closely. The authors sum up that one way of using the device could be a communication tool for expressing emotions between loved ones. 
Chang and O'Sullivan [4] found out in their studies that a mobile phone with tactile feedback had an overall better "feel" to it than a non-tactile phone. They also found a connection between the perceived audio quality and the feel of the phone: devices with a good feel to them are also said to "sound better". This suggests that haptics can be used to improve the overall user experience of a mobile phone.

Regarding user experience, "having an experience" involves various elements, such as personal involvement in the situation (e.g. being interactive in the situation), holistic engagement with a product and very subjective factors (implying one's values and motivations). Kankainen [13] defines user experience (UX) as a "result of a motivated action in a certain context. User's previous experiences and expectations influence the present experience; this present experience leads to more experiences and modified expectations". Hassenzahl \& Tractinsky [10] define UX as "a consequence of a user's internal state (predispositions, expectations, needs, motivation, mood, etc.), the characteristics of the designed system (e.g. complexity, purpose, usability, functionality, etc.) and the context (or the environment) within which the interaction occurs (e.g. organizational/social setting, meaningfulness of the activity, voluntariness of use, etc.)". Hassenzahl [9] also discusses user experience as a holistic perspective, further involving concepts such as joy, hedonism and ludic values, to complement the traditional utilitarian or pragmatic view present in traditional HCI research. Arhippainen \& Tähti [1] complement this by introducing also social and cultural factors as elements that affect the overall experience. Altogether, user experience is seen to be influenced by a multitude of factors, both external and internal (in relation to the user). It is temporally changing, based on user's previous experiences. These versatile and dynamic UX phenomena apply also to haptic communication.

In summary, the research of haptic interaction is often focused on the technologies with which to create various kinds of haptic stimuli. The human factors and user needs for applications utilizing haptic modality for interaction are not yet thoroughly understood. Therefore, we aim to address this gap by studying the potential users' expectations of haptic interaction and applications, especially in the mobile domain.

\section{USER STUDY SETUP}

We conducted qualitative user research to study the expectations related to user experience with haptic mobile communication. Altogether seven focus group sessions in addition to one pilot group were held to understand the users' insight of both the benefits and potential challenges in haptic mobile communication. To compose design implications for future mobile systems using haptics, we identified the most salient factors and users' expectations for the user experience.

\subsection{Research Objectives}

Our main goal was to gain insight into users' expectations regarding the user experience with haptic devices and applications. The focus was on person-to-person communication with mobile devices that is conveyed by the haptic modality. Yet, also other domains, such as navigation and multimedia enrichment, were covered briefly to understand the role of haptics also in other domains. We addressed the issues of the overall factors that affect the user experience with haptics, the needs users have related to haptic communication and how haptic systems could fulfill the aforementioned needs.

\subsection{Methodology}

To gather user data we conducted seven focus group sessions (and one pilot test not included in the results) with both heterogeneous and homogenous groups in two consecutive rounds. Focus groups were selected as a study method because the research subject was future technology that was not available at the time of the study. The method was considered appropriate for the explorative phase of the research. Each focus group lasted approximately 1.5 hours and had 3 to 6 participants. The focus groups are listed in Table 1. The sessions were sound recorded, but the recordings were only used as a backup when the written notes were insufficient. Participants in all of the focus groups were given the same introduction to the topic before the discussions began. The introduction covered the basic characteristics of the sense of touch and some examples of how it could be used in interfaces. Also, some present devices with haptic feedback were quickly introduced.

Table 1: Summary of the focus groups and participants.

\begin{tabular}{llc}
\hline Round & Participant group & Participants \\
\hline & Pilot group & 6 \\
\hline \multirow{3}{*}{ Round 1 } & FG1: Heterogeneous group of students & 3 \\
& FG2: Heterogeneous group of students & 5 \\
& FG3: Heterogeneous group of students & 4 \\
\hline \multirow{5}{*}{ Round 2 } & FG4: People in relationship & 5 \\
& FG5: Outgoing and sporting people & 5 \\
& FG6: Parents & 4 \\
& FG7: Motorists & 4 \\
\hline
\end{tabular}

The discussions in the focus groups were based on scenarios, which acted as a communication-related stimulus material to elicit discussion and let us further inquire the expectations. The scenarios depicted possible use cases for haptics on a general level without specific details in technology or the interaction between user and device. The scenarios were short textual descriptions of possible use cases and contexts for haptic communication (see an example in Figure 1). Scenarios covered communication situations in emotional, leisure and work contexts. In the first round the scenarios were presented on a general level without any precise technological or interaction details in order to gain overall insights into the three mobile application areas for haptics. In the second round our study concentrated only on haptic communication. The level of details in scenarios was slightly increased. This was done to obtain more feedback on initial ideas for prototype development. An overview of the scenarios and themes is presented in Table 2. 
"Sara is going downtown for supper and cinema with her girlfriends. They have all prepared for the night carefully. While waiting for the evening, the girls gather near the city center and decide to go to a nearby park to enjoy the lovely summer weather. The park is full of other citizens too, mainly youngsters spending a summer evening on the lawn. The girls choose a sunny spot near a walkway and sit down.

During the girls' chatter Sara feels her phone move and notices her crush is inviting her to haptic communication. She takes her phone on her palm and squeezes it to answer the invitation. The boy feels how Sara is holding the phone tightly and the touch transfers between the phones. She feels how letters starts to form on the back of the phone... Sara can feel how the boy draws a heart at the end of the message, her phone starts to pulsate and she can't hide her smile. Sara answers to the boy and other girls notice this and the phone in her hand and the conversation changes quickly. They start to playfully tease her about her new crush and marvel at her strange phone."

Figure 1: An example scenario used in focus group sessions

Table 2: Themes of the scenarios

\begin{tabular}{lll}
\hline \multicolumn{1}{c}{ Scenario topic } & Scenario elements & Discussed with \\
\hline Mountain biking & High cognitive load & FG1, FG3 \\
Business meeting & Group, multimodal & FG1, FG2 \\
Missing family & Hugging, emotions & FG3 \\
\hline Motorcycling & Group, signaling a turn & FG5, FG7 \\
Business meeting & Group, multimodal & FG5, FG6 \\
Missing family & Hugging, emotions & FG4, FG6, FG7 \\
New crush & Unimodal, privacy, emotions & FG4, FG6, FG7 \\
Paintball game & Group, unimodal, gaming & FG5, FG7 \\
\hline
\end{tabular}

The scenarios depicted haptic communication situations with high cognitive load, group communication with multiple people, private communication hidden from others, communication of emotional content, and private haptic communication while gaming. Scenarios also had multimodal and unimodal communication contexts. In multimodal situations all modalities were freely usable in addition to haptics, and unimodal situations were restricted to haptic modality only.

The gathered qualitative data was analyzed first group-wise and then scenario-wise. The notes were transcribed and analyzed by qualitative means: categorizing, grouping and abstracting the data, but at the same time, saving the most relevant user quotes and details. These categories and groups are presented in the results. Between-group analysis was omitted, as the groups were found to be too heterogenic despite the different user groups. Moreover, group-wise comparisons between different user-groups were not the focus in this study.

\subsection{Focus Group Participants}

Round 1 of our study was held with heterogeneous groups where participants did not represent any specific application area, whereas the sessions in Round 2 were held with more homogeneous groups with an expected common need for communication technology. Table 1 illustrates the group themes and number of participants in each session. The groups were chosen considering the possible use cases for haptic communication and the scenarios created in advance.

Participants were recruited from various potential user groups to provide different viewpoints. Active use of mobile devices with multimedia features or GPS-navigation, as well as the potential to act in the scenarios were used as the main recruitment criteria. Altogether 30 Finnish users participated in our focus groups, excluding the pilot group. 13 of the participants were female and 17 male. Participants' ages varied from 20 to 44 and the mean age was 27.9 years. A vast majority $(93 \%)$ of the participants were either studying at a university or had a university-level degree. Our background questionnaire had two questions about the level of technical orientation of the participant. These questions and results are presented in Table 3. Our participants were moderately technically oriented as they used technical devices but were mostly not early adopters of new technologies. This focus was intended, as we assumed that slightly technologically oriented participants are open towards the discussion of new technologies and come up with ideas also relating to usage of new means of communication.

Table 3: Background questions about participants' technical orientation and communication habits (1 "I strongly disagree" - 7 "I strongly agree") ( $\mathrm{N}=30)$

\begin{tabular}{lcc}
\hline Question & Mean & St. dev. \\
\hline $\begin{array}{l}\text { "Among my friends, I'm usually first to buy } \\
\text { new devices." }\end{array}$ & 3.9 & 1.9 \\
$\begin{array}{l}\text { "I help my friends and family with the use of } \\
\text { technical devices." }\end{array}$ & 5.9 & 1.1 \\
$\begin{array}{l}\text { "For me, touch is an important part of } \\
\text { communication with others." }\end{array}$ & 4.9 & 1.2 \\
$\begin{array}{l}\text { "I also communicate feelings with a mobile } \\
\text { phone." }\end{array}$ & 5.0 & 1.5 \\
\hline
\end{tabular}

The final two questions presented in Table 3 enquired about the participants' use of touch and emotions in communication. Both questions were rated positively, which shows that among our participants, touch and haptic communication may have potential in addition to the currently used communication modalities. Mobile phones are also presently used to communicate feelings even though the small nuances are often lost.

\section{RESULTS}

In our exploratory study, we gained results about a diverse set of topics related to user expectations of haptic communication. The participants considered haptic communication mostly as an interesting concept but opposing opinions were also expressed. Only a few were either really enthusiastic or very skeptical during the discussion, while the majority thought that adding haptic modality can indeed bring something new to the communication over distance. In the following sections, the results are categorized by the major discussion topics in the focus group sessions.

The initial user expectations that were gathered with the background questionnaire at the beginning of each focus group were rather positive (see Table 4). The questions were part of a background questionnaire in which it was explained that the context of interaction was to be mobile devices. All in all, 
participants believed that using the sense of touch can bring something new into the interaction with mobile devices. Touch was regarded as very natural as an interaction modality. Here, haptic interaction included both output and input modalities. Despite the quite positive expectations, participants expected they would not use haptics very often.

Table 4: Participants' initial expectations at the beginning of the focus group sessions. (1 "I strongly disagree" - 7 "I strongly agree") $(\mathrm{N}=30)$

\begin{tabular}{lcc}
\hline Question & Mean & St. dev \\
\hline $\begin{array}{l}\text { "I believe using the sense of touch brings } \\
\text { added value to interaction." }\end{array}$ & 5.6 & 1.1 \\
$\begin{array}{l}\text { "I believe interaction based on touch is } \\
\text { natural." }\end{array}$ & 5.7 & 1.1 \\
$\begin{array}{l}\text { "I would often use touch-based feedback." } \\
\text { "The idea about touch-based interaction with } \\
\text { mobile devices feels strange." }\end{array}$ & 4.7 & 1.2 \\
\hline
\end{tabular}

\subsection{Challenges in Current Communication - an Opportunity for Haptic Interaction?}

Before studying participants' expectations for haptic interaction we briefly inquired about the challenges with currently used modalities in communication over distance as well as mobile technologies. The aim was to identify new possible use cases for haptic interaction in communication. This discussion also served as a basis for the following discussion about when haptic interaction would be preferable.

Our participants recognized and expressed the general limitations of current mobile devices. Audible and visual feedback was considered not to be always enough because of the varying and demanding contexts in mobile communication. Audible modality was not seen as socially accepted in certain contexts and not very well recognizable in noisy environments. Visual feedback was considered to draw the user's attention from other - perhaps more important - tasks. Moreover, participants pointed out that they want to communicate in public places, such as shopping centers and on public transportation, without disturbing others and revealing private information publicly. Even text messaging was regarded as too disturbing in certain situations. The participants agreed that these limitations could, however, be at least partially avoided by representing the information via the tactile modality.

The difficulty of interpreting emotions in communication was brought up in several focus groups. The participants commented on the fact that certain emotions and attitudes, such as sarcasm and humor, are difficult to convey and understand from text messages - and also to some extent from phone calls. We regard these as consequences of the unimodal (haptics only) nature of interaction, which discards the non-verbal, often subconscious, messages. The lack of these non-verbal nuances was stated as easily leading to misinterpretations even in communication with close friends and acquaintances. These misinterpretations can lead to negative experiences and thus lower the perceived user experience of current mobile phones. In addition, the participants stated that in emotional situations the right words are sometimes difficult to find and even unnecessary. Despite the challenges, most of the participants use these means of communication to convey emotive content (see Table 3).
Participants reported that they find it difficult to maintain their concentration on the textual or verbal-only communication. The added effort to maintain concentration during long conversations with either modality makes communication more exhausting than in a face-to-face situation. This leads to other activities during the communication and parts of the messages can be easily ignored. As an implication, participants wanted ways to intensify and enliven the present communication e.g. making the involvement easier and adding more ludic elements. Some also commented that such enlivenment would increase the feeling of presence and closeness of the communicating parties.

Many participants found the lack of responses a fundamental problem of communicating over a distance. Thus, a way to receive instant feedback from the recipient was desired. Sharing one's current status with others was mentioned as a tool for this problem. The ability to inquire about it at any given time would give hints to the user when the other end is not responding. The status messages would provide an insight into one's current activities and aid the decision of when to communicate or why the recipient is not answering. Participants brought up poking and knocking metaphors to inquire about their contacts' statuses and gain their attention.

\subsection{Needs and Expectations for Haptic User Experience}

Overall, the scenarios with simple binary information information that can be expressed with a single word e.g. yes/no answers - or emotional content sometimes difficult to express verbally, such as longing or affect, were seen as the most suitable for haptic unimodal communication. This is nicely elucidated by the user quote below.

\section{"Sending hugs would really work in this situation." \\ - Focus group 7}

When other modalities are added, haptics could be used more versatilely. Scenarios with group communication or more complex exact information were not liked as the information would become difficult to interpret. Multiple contacts communicating simultaneously and indentifying senders in these situations were expected to be confusing. For exact information, such as telling the time, the participants felt the unimodal haptic interaction too limited and vague. Complex messages would require agreements between users which were mostly unwanted. Few expected that haptics could be only used to grab the recipient's attention because of this limitation.

The main benefit of haptic communication was seen to be the added richness and immersion it can potentially bring to communication, which can increase the personal involvement the experience. It could become more holistic with regard to the level of interaction and thus richer information could be conveyed. Another prominent benefit was regarded to be the spontaneity and quickness of communication. Haptic communication was seen to be an easy, simple and fun way to convey sudden emotions. Therefore, the quickness of creation was emphasized as emotions often change or fade out quickly: it was seen as a way to reduce the delay between the emergence of the emotion and acting accordingly. Being quick to create and perceive haptic messaging would be especially appropriate in the mobile context. For example, a haptic response could be sent to quickly inform 
somebody that a message has been received or agreed. This is essential in mobile contexts, where attention is often needed for multiple tasks.

The quickness is also partly based on the pervasive nature of the haptic stimuli: participants agreed that it is easy for people not to hear but difficult not to feel. Unimodal haptic messages could also be used to send emotive messages just to remind someone that "I'm thinking of you". Yet, it was brought up that even the haptic modality can not replace actual closeness and intimacy between individuals even though it can add some elements of these to communication. The remote communication would still lack elements of the face-to-face experience (e.g. odors and sensation of closeness).

\subsubsection{Expectations for Haptic Interaction}

One of the most important discussion topics was the physical interaction between the user and the communication device. With regard to most common communication situations, haptic stimulus as part of a multimodal message was preferred to unimodal haptic messages. Participants often mentioned that haptic feedback could be best used to support currently available modalities in communication. As contexts of communication can rapidly change in mobile applications, multimodal communication can also convey information about the context. Haptic stimulus was seen to be able to add and augment the currently missing nuances and ease the use of mobile devices in difficult situations. For example, to convey a mood, a haptic input could either support the multimodal message as haptic output or be mapped to visual modality (e.g. represented in colors). Unimodal interaction was perceived mostly as imprecise and suitable for conveying only small amounts of information or emotions.

The most promising way to create haptic information was seen to be mimicking the real-life communication situations. Instead of a menu-based approach, touch-based messages should be created by the same modality by touching the device in various ways or by making gestures with it. The participants regarded fluency of communication and the added privacy haptics brings by making communication and its content hidden as important. Similar ways of touching and gesturing were suggested with the device as would be used in face-to-face situations.

Stroking, poking and shaking were seen as the best ways to interact with the device. Some participants suggested drawing symbols on the device. This was seen as a very close and intimate way of communication, resembling drawing symbols on the receiver's hand, for example. All in all, the participants emphasized that the interaction should be such that the receiver would feel the created haptic messages in a similar way they were created, preferably with short delays. Participants also acknowledged this is a difficult technical challenge. Tactile feedback, such as vibration, touch-like pressure and temperature changes were mentioned as the most noticeable and humane ways to receive feedback from the device. Also, pinching, stretching and electrical output were mentioned, but soon discarded as impractical or otherwise inconvenient by the participants.

Dynamic input and output were common parameters for all different interaction types. Participants strongly emphasized that the device has to be able to sense and display the varying intensities of touch. Emotions have different subjective energy levels and transferring these is essential for bringing the intended richness to communication. Gestures from a gentle and soft stroking to angry shaking should not be limited by the used technologies. This supports earlier findings where too limited expression is said to ultimately lead to withering (cf. [1]). Creating a framework which supports rich communication and the creation of users' own haptic language can make it more sustainable. The participants projected that a new kind of language or symbolism could evolve when people learn to use the modality that has traditionally been ignored in remote mobile communication. For example, people could create a set of haptic emoticons, where each emoticon would represent a certain emotion or even informative content.

Our participants noticed the same synchronic and asynchronic communication methods as Chang et al. [4]. Both call-like (continuous, asynchronic) and message-like (discrete, synchronic) features were desired. The participants saw that continuous interaction would allow playful touch-like interplay similar to real touching. The discrete communication would make it possible to save and "play" messages when appropriate - a feature many participants expected to be found in a mobile device. Yet, being highly related to the context of communication, asynchronic communication was seen to bring more added value and new possibilities to conveying emotions in communication. Basically, the situation and content of the communication were seen to determine which type of interaction would be used.

One special doubt in mobile haptic communication was anticipated as to whether the message was actually received or not. As the amount of information in haptic modality is limited, the design of a feedback method for communicating this must be very delicate. Related to this, some participants also wanted to know why the other end is communicating with haptic modality instead of using a phone call or other more common means of communication. The context of communication (why the tactile response is given) should be able to be expressed by other means (e.g. by speech, text or images).

\subsubsection{Information Content in Unimodal Haptic Communication}

Considering the information content, unimodal haptic communication was regarded to be most promising in conveying emotions and moods in situations where words are not necessary. In respect of unimodal haptic communication, the participants saw that emotive and very private messages could be sent, for example, between partners, families and close friends. In addition, emotive haptic communication was seen as two-way and reciprocal. Participants felt that one should respond to a haptic message with another haptic message. Haptic communication represents the most private and intimate remote communication, as is emphasized in the following user quote:

$$
\begin{gathered}
\text { "An SMS would seem emotionless after a warm haptic } \\
\text { message." - Focus group } 4
\end{gathered}
$$

The difficulty of expressing precise information may limit the possible use scenarios. The unimodal emotive messages could be misinterpreted without any additional information and would require very good knowledge of the sender. Due to the limited capability of coding information in haptic modality, conveying exact and factual information merely with the haptic modality was not considered reasonable. Too detailed factual information would 
require users to agree and learn complex patterns or completely new languages e.g. Morse code. Participants gave some examples of possible uses for unimodal communication - initiating other means of communication via haptic modality and drawing the receiver's attention. The participants saw that the information has to be tied to context to be interpretable. Both the sender and receiver must have a common understanding of the context - a common convention of haptic language. Some participants anticipated that haptic communication would require a close relationship in order to have this common understanding. The closer the sender is to the recipient, the easier the message would be understood merely as unimodal, and the more likely haptic communication would be used in the first place. Moreover, the level of comprehensibility was seen to also depend on how much this haptic way of communication has been used before between the communication partners. This consolidates the discussion in linguistics about a concept of common ground: a shared basis of understanding that serves as a platform for the interaction (see e.g. [6]). Then, by offering a few easy-to-understand options, such as binary information (e.g. agree/disagree), even very exact information could be conveyed.

\subsubsection{Privacy and Wearability Affecting the User Experience}

The overall quality of the haptic stimulus itself largely determines how the user experience of haptic communication appears: it affects physical sensations (e.g. involvement, arousal and evocation), information content that can be conveyed by it (e.g. factual and emotive), overall functionality and purpose as well as the feeling of closeness with the other end in the communication situation. Nevertheless, other elements affecting the overall user experience also came up in the focus group sessions.

The participants emphasized that they need to control the privacy of haptic communication more than with other communication channels. Touch was regarded as such a private sense that the user must be able to control who can communicate via the haptic modality with them. This should be the case even when touch is conveyed through technology. This ability to control contacts would work as a metaphor for personal space. Basically, haptic communication was said to be only used with one's spouse or very close friends and family, as illustrated in the user quote below. This was partly due to the emotional information that would be conveyed, and partly due to the pervasiveness and depth of the interaction.

\section{"I would definitely restrict the individuals who can use haptic modality in communication with me, and I have probably less than 10 of them." - Focus group 6}

Few participants noted that mobility together with gesture input can create privacy problems in communication applications. Movement with large trajectory or speed is not suitable in public, if users want to hide the communication or its contents. Instead, participants said they would prefer to use small and unnoticeable ways to create messages such as squeezing, tapping or touching. They were also concerned about how they would appear to others if they are making gestures in public.

Moreover, haptic communication was regarded as one-to-one. Group communication situations did not come into question because of the nature of communicated information. Also, the participants anticipated that the physical interaction could become too challenging because identifying different senders and partly temporally overlapping messages would be difficult, especially with unimodal communication.

Wearability and the location of the tactile feedback came up regularly. A watch-like device worn on wrists was most often suggested.

\section{"The message could be received in some kind of bracelet" - Focus group 1}

It would be unobtrusive, mobile and constantly in contact with the user's skin while leaving both hands free for other activities. Actuator placement at the wrist has been previously studied and found to be feasible. A total of 4 actuators, or even more with training can be identified on both sides of the wrist [5]. Hand-held haptic devices, often a part of or a feature in future mobile phones were also mentioned. However, it was not the preferred design for many as it needs to be actively held in the hand when used. In contrast to these rather small devices, some preferred a more comprehensive shirt-like device capable of producing tactile feedback in the whole torso. This would allow more extensive and immersive experience while also leaving the hands free. However, it should be transferrable or included in all clothes. Perhaps this led to the idea of "tactile stickers", freely placeable tactile feedback elements. Head-mounted actuators were mentioned a few times in the focus groups, but this was a very sensitive topic. Few participants suggested head-mounted devices, but when we introduced this idea to other groups the feedback was negative. Perhaps they were thinking about present vibrating actuators mounted on their head or affecting their appearance in general, which led to a negative image.

"Head mounted things in general seem to be difficult for people." - Focus group 2

Some participants were truly fascinated by the possibility of conveying touch over distance, while some were afraid it might open new ways for bullying and harassment or it might alienate people from face-to-face communication.

This new modality is suited to playful and fun ways of communication with touch and as such we see it primarily as a hedonistic addition to current remote communication applications.

\section{DISCUSSION}

Six design guidelines were formed from the results presented above. The focus is on designing good user experiences in mobile communication, although the principles may also apply to the design of other applications enriched with haptic information.

Our participants felt that current mobile communication devices are limited. Communication is restricted to one modality only and as a result lacks richness. This makes the communication harder to interpret and more exhausting during longer conversations. Participants liked the ideas embodied in emotive haptic communication and simple binary information. The main advantages over the present communication methods were added richness, privacy and spontaneity of communication. For unimodal interaction these were the preferred use cases, but more complex information did not feel impossible if the future implementations are expressive enough. Haptic stimuli can also be used to enhance present communication modalities, thus bringing more use possibilities. 
Haptic communication was seen as the most intimate way of communication, which is shared only with the closest contacts. For the user experience, the quality of the haptic stimuli and the resulting sensations were the major factors. Moreover, the physical design of the device affects the affordances. A handheld device was not the preferred form factor for all as it needs to be actively held and requires at least one free hand.

\subsection{Design Guidelines for Haptic Mobile Communication}

Although based on a rather narrow study, we encourage following the design implications when designing haptic communication applications in mobile devices. By taking the various aspects the implications cover into account, we believe it will facilitate the adoption of this new technology as well as create new unique communication possibilities. Hence, a satisfying and rich user experience could be achieved.

\section{Provide a clear interaction model between the user and the application.}

Currently, people do not have much previous knowledge or experience as to how to interpret artificial haptic stimuli created by mobile devices. Because of the low-informative nature of haptic stimuli, the interaction model with unimodal haptic applications must be very clear. Firstly, design haptic applications with clear mappings between users' actions and the generated output stimulus. Clearly indicate when haptic stimuli are being generated and sent to prevent accidental communication when the mobile devices are handled. Secondly, the incoming haptic communication stimuli must be distinguishable from other tactile signals from the device. In asynchronous communication the incoming and outgoing signals must be separated, if the feedback from the outgoing stimuli is presented to the user.

Overall, the abstraction of touch to artificial stimuli must match the expectations of the users to make it intuitive and allow spontaneity. People have a good instinct in regard to the sensations we get and create when touching in real-life. While designing, these experiences and expectations should be taken into account. For example, the stimuli must be dynamic enough to be able to express the large variety in both intensity and the extent of people's touch.

\section{Minimize the amount of information in communication.}

Haptic interaction was found to be most appropriate in emotive communication situations. The human touch sensory system is not as absolute and refined as the visual or auditory senses. Hence, the information content conveyed by haptic modality might be very ambiguous and vague to the users. Due to these facts, users can become confused with too complex tactile feedback at both the receiving and sending end. Avoid applications where it is necessary for exact information to be conveyed, as it is not suitable for unimodal haptic communication. Information incoming from multiple sources at the same time was not regarded as acceptable. Therefore, remote communication would perform best in one-to-one situations. In group communication situations it would be challenging to notify who the sender is and to choose who the recipient should be. The one-to-one communication could, for example, be implemented as a "hot line" that is constantly open between the contacts once the recipient is selected. Here, the recipient would be always known and confusion would be avoided.
Support the spontaneity of touch.

Applications that need constant attention are difficult or even dangerous to use while mobile. Creating and receiving haptic stimuli must allow spontaneous communication without browsing deep menus or pressing multiple buttons. Interpretation of touch is time and context dependent. Therefore, as in real life, using touch in mobile communication should be straightforward and natural without unnecessary complexity in the user interface. Ideally, creating touch through a haptic application should not inflict any more cognitive load than creating a real touch. As the amount of exact information is limited, mobile haptic communication should focus on benefits the other modalities do not have, and spontaneity must be one of them.

Spontaneity can be supported, for example, by providing features for directly creating and manipulating haptic input that would be received in real-time. This would open new kinds of opportunities for instant messaging between people with close relationships. Secondly, spontaneity can be supported by providing possibilities to reply to textual or other kind of messages with simple haptic messages (e.g. agreeing to a suggestion) instead of using the same modality as in the first message.

\section{Provide both asynchronous and synchronous communication} methods.

As the current communication situations are so mundane and diverse, people appreciate the possibility to use both synchronous and asynchronous ways of communicating in current mobile phones. People have created personal habits for communicating different types of information with these various means. Hence, the design should let the users choose freely between asynchronous call-like and synchronous message-like communication methods based on their preference and the context of communication. As the mobile communication situations vary, both continuous and discrete methods of communicating should be supported.

The asynchronous method was considered best to communicate emotions, while the synchronous method can convey small amounts of information like agreements. The asynchronous methods should enable users to dynamically create, and at the same time receive, haptic information to allow continuous interplay, which best mimics the immediacy of face-to-face communication. This can be done unimodally as well as multimodally along with other communication channels. The asynchronous interaction could be, for example, stroking, drawing symbols and letters with one's fingers or making slight gestures with the device. The message should be mediated "as it is", i.e. the device usually should not try to interpret the meaning of the message but send it as such. The synchronous model could be implemented to transfer small messages such as "Are you available?", "Let's meet tonight" or "OK". Such messages with a small amount of information can also be understood later on, outside of the communication context, and maybe are not as urgent.

Provide features for the creation of a new haptic symbolism. In order to survive and become a reasonable way of communicating, haptic mobile applications must allow expressive ways of communicating. Support users' creativity in creation and interpretation of haptic messaging and our ability to interpret the meanings of others' messages. Provide a rich way to create and edit haptic information instead of limited and pre-defined 
templates. To create messages with dynamic content, directly touching or performing gestures by moving the device should be supported.

Providing a rich means to create haptic messages can lead to the emergence of a haptic symbolism or language, where certain stimuli have developed during the evolution of the interaction. The emerged symbolism would provide common ground for those who are communicating with haptic applications. The basic elements of the symbolism could even become universally known. Corresponding phenomenon have been observed in the emergence of emoticons and partially also in SMS communication.

\section{Let the users control access rights.}

Provide users with a way to grant specific contacts a right to use touch as a communication channel. In real life, touch is a very private and intimate way of expression. Messages mediated by touch involve emotional information that is not shared with everybody. The access rights control could be implemented by allowing users to group their contacts, for example. This grouping could be used to form an "inner circle" of the closest contacts for which the use of haptic modality is freely available. Grouping should be a two-way agreement between the users and the application should preferably provide an easy way to initiate, view and manage these agreements. Furthermore, the user could be able to determine modes, in which she would be accessible via the haptic modality.

\subsection{Revising the Study}

Inquiring about expectations for user experience is challenging when no functional prototype is available and thus no actual and physical interaction can be tested. This is especially challenging when exploring such a tangible and concrete type of interaction as haptics. Hence, discussing and letting participants describe their own needs and expectations does not fully replace observing them in a real context with a functional device. They might not be able to indentify and express their true and possible needs and expectations precisely. This is further affected by the novel and private nature of the theme - some topics might be too private to share in a group or they are simply difficult to comprehend in such a limited time. The expectations are based on a limited number of scenarios and thus, the results can not be generalized completely. The introduction materials inevitably affected the users' conception of haptics. However, the first expectations provide a basis for the upcoming research and development. In spite of the challenges, we regard this study to be an important move towards aspiring to understand the users' expectations even before developing haptic communication applications.

The theme of our study was physical by nature and therefore difficult to discuss based only on visual and auditory stimulus (scenarios and introduction). It was challenging to present the topics to the participants without giving too specific examples as the purpose was to study their expectations (not just to evaluate existing designs). Due to the short and lightweight introduction, the participants might have had varying or misled conceptions of the possibilities and limitations of haptic communication. However, we believe it is useful for the early phases of the design process to gain understanding of the potential users' expectations and concerns. This can be used to inform design phase and to ensure the enjoyable user experience. Later on, the topics can be further studied with prototype for example.
The participant selection becomes important, as the study was not extensive enough to be able to generalize the findings to larger populations. The participants were highly educated, Finnish and from a limited age group. Moreover, different cultural backgrounds inevitably affect how people communicate and how they share personal information, such as emotions. The actual effects of the participant selection remain unknown to us without further research.

\section{CONCLUSIONS AND FUTURE RESEARCH}

The main contribution of the study is the new knowledge about the expectations and user experience of potential users of haptic communication systems. We gained insight into the expectations towards the holistic user experience with haptic systems with mobile devices. To summarize our findings, we drew up design implications for haptic communication systems. The results can serve as a fruitful basis for future studies of the haptic user experience and the related scientific discourse, as well as for designing the first prototypes of haptic mobile communication systems.

Our focus group study shows that haptic modality has potential in conveying emotions and binary information in one-to-one communication. Users found a use for both synchronous and asynchronous communication. However, the emphasis was on the latter and continuous communication, which can add non-verbal hints to the remote communication desired by the participants. This requires a haptic language the users can understand and designers should provide users with applications that are rich and expressive in communication. To make it fluent, the interaction itself should resemble or have similar metaphors as everyday use of touch sensation. Ease of use, the subjective quality of haptic stimulus and the ability to convey rich, even non-verbal messages, were the most relevant factors regarding user experience. The issue of privacy created a discussion between our participants touch is not shared with strangers or even with all friends. Moreover, the added privacy haptic communication can bring was seen as beneficial for remote communication.

The participants' comments and reactions suggest that haptic communication is seen as more playful and fun compared to the present textual and auditory alternatives. Participants who naturally expressed themselves easily by using body language were genuinely enthusiastic about the idea. While the alternatives offer a familiar way of conveying exact information, haptic communication can convey emotions and feelings that can be difficult to put into words. However, the expressiveness of haptic modality appears to be the biggest challenge in real-life communication and it must be studied more carefully. Conveying exact information was seen very difficult. Many expected that the content of haptic messages should be agreed beforehand to be fully understandable.

In our study we have not yet discovered all the factors affecting the user experience in haptic communication. Our ongoing research will focus next on 1) creation and interpretation of unimodal haptic messages (related to the development of "common ground"), 2) combining haptic information to multimodal communication, and 3) studying more carefully what kind of content is suitable to be conveyed via haptic modality. We will conduct field studies to evaluate the user experience of a 
prototype mobile device that has been designed based on our design guidelines. We anticipate gaining diverse results from the evaluation of actual prototypes as they will demonstrate and concretize many issues that the focus group participants might have understood or postulated incorrectly.

\section{ACKNOWLEDGEMENTS}

We would like to thank Teemu Ahmaniemi, Vuokko Lantz, Roope Raisamo, Veikko Surakka, Jukka Raisamo, Jussi Rantala, Jani Lylykangas, Outi Tuisku and Kaj Mäkelä for their insightful comments in designing the study and analyzing the results.

\section{REFERENCES}

[1] Arhippainen, L., Tähti, M. 2003. Empirical Evaluation of User Experience in Two Adaptive Mobile Application Prototypes. Proceedings of the 2nd International Conference on Mobile and Ubiquitous Multimedia, Norrköping, Sweden

[2] Brave, S. \& Dahley, A. 1997. In Touch: a medium for haptic interpersonal communication. In CHI '97 Extended Abstracts on Human Factors in Computing Systems: Looking To the Future, Atlanta, Georgia, March 22 - 27, 1997. ACM PRess, New York, NY, USA, pp. 363-364.

[3] Brown. L. M., Williamson, J. 2007. Shake-to-talk: Multimodal Messaging for Interpersonal Communication. In Proceedings of the 2nd International Workshop on Haptic and Audio Interaction Design (Seoul, Korea). Spinger LNCS.

[4] Chang, A., O'Modhrain, S., Jacob, R., Gunther, E., and Ishii, H. 2002. ComTouch: design of a vibrotactile communication device. In Proceedings of DIS 2002, London, England, June 25 - 28, 2002. ACM Press, New York, NY, USA, pp. 312320.

[5] Chen, H.-Y., Santos, J., Graves, M., Kim, K., and Tan, H. Z. 2008. Tactor localization at the wrist. M. Ferre (Ed.): EuroHaptics 2008, LNCS 5024, pp. 209-218.

[6] Clark, H. H. 1996. Using Language. Cambridge, UK: Cambridge University Press.

[7] Fukumoto, M. and Sugimura, T. 2001. Active click: tactile feedback for touch panels. In CHI 'O1 Extended Abstracts on Human Factors in Computing Systems, Seattle, Washington, March 31 - April 05, 2001. ACM Press, New York, NY, USA, pp. 121-122.

[8] Haans, A., de Nood, C., and IJsselsteijn, W. A. 2007. Investigating response similarities between real and mediated social touch: a first test. In $\mathrm{CHI}$ '07 Extended Abstracts on Human Factors in Computing Systems, San Jose, CA, USA, April 28 - May 03, 2007. ACM Press, New York, NY, USA, pp. $2405-2410$.

[9] Hassenzahl, M. 2003. The thing and I: understanding the relationship between user and product. In M. Blythe, C. Overbeeke, A. F. Monk, \& P. C. Wright (Eds.), Funology: From Usability to Enjoyment. Kluwer Academic Publishers, Dordrecht, Netherlands, pp. 31-42.

[10] Hassenzahl \& Tractinsky. User Experience - a Research Agenda. Behaviour and Information Technology, Vol. 25, No. 2, March-April 2006, pp. 91-97.
[11] Hoggan, E. \& Brewster, S. 2007. Designing audio and tactile crossmodal icons for mobile devices. In Proceedings of ICMI 2007, Nagoya, Aichi, Japan, November 12 - 15, 2007. ACM Press, New York, NY, USA, pp. 162-169.

[12] Hoggan, E., Brewster, S. and Anwar, S. Mobile MultiActuator Tactile Displays. To appear in Proceedings of 2nd International Workshop Haptic and Audio Interaction Design (Seoul, Korea). Springer LNCS.

[13] Kankainen, A. (2003). UCPCD: user-centered product concept design. In Designing For User Experiences, Proceedings of the 2003 conference on Designing for user experiences (pp. 1-13). New York: ACM Press.

[14] Leung, R., MacLean, K., Bertelsen, M. B., and Saubhasik, M. 2007. Evaluation of haptically augmented touchscreen gui elements under cognitive load. In Proceedings of ICMI 2007, Nagoya, Aichi, Japan, November 12 - 15, 2007. ACM Press, New York, NY, USA, pp. 374-381.

[15] Linjama, J. \& Kaaresoja, T. 2004. Novel, minimalist haptic gesture interaction for mobile devices. In Proceedings of NordiCHI 2004, Tampere, Finland, October 23 - 27, 2004. ACM Press, New York, NY, pp. 457-458.

[16] Luk, J., Pasquero, J., Little, S., MacLean, K., Levesque, V., and Hayward, V. 2006. A role for haptics in mobile interaction: initial design using a handheld tactile display prototype. In Proceedings of the SIGCHI Conference on Human Factors in Computing Systems, Montréal, Québec, Canada, April 22 - 27, 2006. R. Grinter, T. Rodden, P. Aoki, E. Cutrell, R. Jeffries, and G. Olson, Eds. ACM Press, New York, NY, USA, pp. 171-180.

[17] MacLean, K. E. 2000. Designing with Haptic Feedback. In Proceedings of ICRA 2000, San Francisco, CA, USA, April $22-28,2000$.

[18] Merriam-Webster Online Dictionary. 2008. [WWW]. [Cited 26/1/2009]. Available at: http://www.merriamwebster.com/dictionary/haptic.

[19] Mortensen, C. D. 2007. Communication Theory. $2^{\text {nd }}$ edition. New Jersey, USA. Transaction Publishers.

[20] Oulasvirta, A., Tamminen, S., Roto, V., and Kuorelahti, J. 2005. Interaction in 4-second bursts: the fragmented nature of attentional resources in mobile HCI. In Proceedings of the SIGCHI Conference on Human Factors in Computing Systems, Portland, Oregon, USA, April 02 - 07, 2005. ACM Press, New York, NY, USA, pp. 919-928.

[21] Pakkanen, T., Lylykangas, J., Raisamo, J., Raisamo, R., Salminen, K., Rantala, J., and Surakka, V. 2008. Perception of low-amplitude haptic stimuli when biking. In Proceedings of ICMI 2008, Chania, Crete, Greece, October 20 - 22, 2008. ACM Press, New York, NY, USA, pp. 281-284.

[22] Smith J., MacLean K. 2007. Communicating Emotion Through a Haptic Link: Design Space and Methodology. In International Journal of Human-Computer Studies. Volume 65, Issue 4, April 2007, pp. 376-387.

[23] Yohanan, S., Chan, M., Hopkins, J., Sun, H., and MacLean, K. 2005. Hapticat: exploration of affective touch. In Proceedings of ICMI 2005, Torento, Italy, October 04 - 06, 2005. ACM Press, New York, NY, USA, pp. 222-229. 